\title{
Deep Brain Stimulation in Multiple System Atrophy Mimicking Idiopathic Parkinson's Disease
}

\author{
Navamayooran Thavanesan ${ }^{a} \quad$ Martin Gillies $^{b} \quad$ Michael Farrell $^{c}$ \\ Alex L. Green ${ }^{\mathrm{b}}$ Tipu Aziz ${ }^{\mathrm{b}}$ \\ ${ }^{a}$ Queen Alexandra Hospital, Portsmouth, and ${ }^{b}$ Department of Neurosurgery, John \\ Radcliffe Hospital, Oxford, UK; ${ }^{C}$ Department of Neuropathology, Beaumont Hospital, \\ Dublin, Ireland
}

\section{Key Words}

Deep brain stimulation · Multiple system atrophy · Idiopathic Parkinson's disease

\begin{abstract}
Deep brain stimulation (DBS) is approved for idiopathic Parkinson's disease (IPD) but has a poor evidence base in Parkinson-plus syndromes such as multiple system atrophy (MSA). We describe the clinical and neuropathological findings in a man who was initially diagnosed with IPD, in whom DBS was unsuccessful, and in whom MSA was unexpectedly diagnosed at a subsequent autopsy. This case report highlights that DBS is often unsuccessful in MSA and also demonstrates that MSA can masquerade as IPD, which may explain treatment failure in a small group of patients apparently suffering from Parkinson's disease. Additionally, it also presents a case with an unusually long duration of disease prior to death, comparable only to a handful of other cases in the literature.

(c) 2014 S. Karger AG, Basel
\end{abstract}

\section{Introduction}

Multiple system atrophy (MSA) comprises a group of disorders which display differing degrees of parkinsonism, cerebellar abnormalities, corticospinal degeneration and autonomic dysfunction. Pathologically, it is characterised by glial cytoplasmic protein inclusions such as hyperphosphorylated $\alpha$-synuclein. MSA and idiopathic Parkinson's disease (IPD) overlap clinically; DOPA-responsive symptoms, for example, are seen in a significant percentage of MSA patients. Deep brain stimulation (DBS) is approved for IPD; however, its role in managing Parkinson-plus syndromes is less promising, with published 
Thavanesan et al.: Deep Brain Stimulation in Multiple System Atrophy Mimicking Idiopathic Parkinson's Disease

clinical experience of its use in MSA limited to case reports to date. We report the clinicopathological findings of a patient with a 19-year history of DOPA-responsive parkinsonism typical of IPD who failed DBS therapy and in whom MSA was unexpectedly confirmed histologically at autopsy 6 years after surgery, supporting current literature regarding DBS in MSA.

\section{Case Report}

\section{History and Examination}

The patient was a 46-year-old male diagnosed with early-onset IPD at 37 years of age, 10 years after initial symptom onset. Originally, his symptoms consisted of tremor in the left hand which subsequently progressed bilaterally. He was started on Sinemet, Madopar, and pramipexole. Symptoms remained mild and DOPA responsive, with little incapacitation until the age of 40 when there was clinical deterioration including marked bradykinesia, rigidity, stammering, hypophonia, gait freezing, erectile and bladder instability. The patient had a family history of IPD.

On examination, the patient demonstrated a stiff, non-festinating, narrow-based gait lacking arm swing with a dragging left foot, slow turning and gait freezing whilst off medication. There was no gait ataxia. Brisk reflexes and down-going plantars were noted, but postural instability and hypotension [Unified Parkinson's Disease Rating Scale (UPDRS) score 0 off medication] were not evident and Romberg's test was negative. Pill-rolling tremor was present at rest and worse on holding posture, but he was able to sip water without spillage with both arms. There was no evidence of past-pointing or nystagmus. He did not have dystonic features and was not dysarthric (hypophonic tremulous voice improved with L-DOPA). Whilst on medication, he showed marked improvement indicating clear DOPA-responsive asymmetric disease (UPDRS score dropped from 58 to 32). His Hoehn and Yahr score was 2 in both the on and off states. Autonomic dysfunction was not seen on initial presentation. He had a single episode of noted postural drop, associated with an episode of inflammatory colitis. Preoperatively, however, he experienced urinary symptoms and erectile dysfunction (urodynamics later confirmed an overactive bladder causing urgency and incontinence).

He was assessed and accepted for DBS at the age of 41 , approximately 4 years after presentation, on the basis of symptomatic progression, needing maximal medication doses, dyskinesias and on-off phenomena. Neurocognitive assessment was broadly average except for memory, which was below average. When re-examined a year later just prior to surgery, his neurology had deteriorated further; however, his DBS protocol MRI performed immediately before surgery was reported as normal; specifically, there was no hot cross bun sign in the pons or reduction in the putaminal T2 signal relative to globus pallidus (fig. 1). No functional imaging was conducted. His examination features were consistent with parkinsonism; however, brisk reflexes, including jaw jerk, and clonus were also noted. There were no distal limb contractures. Bilateral DBS of the subthalamic nuclei (STN) was carried out in 2 stages in May 2008.

\section{Postoperative Course}

Stage 1 initially gave positive results barring an episode of confusion, hypotension and reduced Glasgow Coma Scale (GCS) score attributed to overstimulation. Titration was effective, and he was switched from regular Madopar to PRN. Stage 2 was complication free aside from transient calibration paraesthesia. Medications were stopped 2 days post- 
operatively with good gait, no tremor, no freezing and improved left arm mobility. The clinical nurse specialist and the patient specifically reported improvement in rigidity and gait. The patient was discharged 3 days later.

The patient subsequently deteriorated after 2 weeks, returning to pre-operative medication doses, with his symptoms worse now than preoperatively. He experienced confusion, short-term memory impairment and hypertonia in the left arm. Subsequent titration helped minimally. In the subsequent 4 years until the time of his death, the patient developed episodes of psychosis, dopamine dysregulation syndrome, IgA nephropathy, chronic renal failure and ultimately terminal Clostridium difficile colitis.

\section{Neuropathology}

Neuropathological examination, performed 6 years after surgery, confirmed the placement of DBS electrodes in the STN (fig. 2). The neuropathological findings were those of MSA. The pathological diagnosis was based on extensive striatal and subcortical white matter accumulation of $\alpha$-synuclein coupled with profound depletion of the neurons from the striatum and substantia nigra with atrophy and gliosis. Oligodendroglial $\alpha$-synuclein inclusions were also very extensive in cerebral and cerebellar white matter, and the middle cerebellar peduncle also showed prominent $\alpha$-synuclein-positive glial inclusions (fig. 3) indicating elements of both a striatonigral and olivopontocerebellar pattern of distribution. Noteworthy was the finding of prominent $\alpha$-synuclein-positive glial inclusions adjacent to the DBS insertion site in the STN.

\section{Discussion}

\section{Diagnosis}

This young male diagnosed with early-onset IPD failed to respond to DBS carried out for rapid clinical deterioration, which culminated 19 years after disease onset. The clinical diagnosis was appropriate based on his responsiveness to L-DOPA (reflected in improved UPDRS scores), the absence of cerebellar signs, abnormal eye movements, orthostatic hypotension or limb weakness, and a normal brain MRI. Prior to surgery, there were significant urinary and sexual problems; however, these are not unusual in advanced IPD.

Diagnosis of MSA is clinical; however, this case demonstrates that in the early phase of its pathology, MSA is clinically similar to IPD. The primary discriminator is usually the lack of a sustained dramatic DOPA responsiveness, as seen in IPD, while additional signs include the presence of autonomic, ataxic or pyramidal features (features of MSA). A clinicopathological review of 203 MSA cases, however, reported that $87 \%$ of cases demonstrated parkinsonism, $74 \%$ experienced autonomic dysfunction, 54\% had cerebellar ataxia, $49 \%$ had pyramidal signs, and 28\% were showing improvements on L-DOPA [1]. A subgroup of patients with MSA is therefore clinically indistinguishable from IPD in presentation and clinical features.

\section{Role of DBS in MSA}

The role of DBS in MSA has not been specifically studied in trials; thus, data consists primarily of case reports (level 3 evidence). The consensus is that patients with a Parkinsonplus syndrome are not suitable DBS candidates for parkinsonian symptoms. Several authors [2-10] have described similar cases where incorrect preoperative IPD diagnoses showed positive initial responses to surgery followed by a rapid decline secondary to progression of MSA and subsequent death or severe disability from complications of MSA (table 1). Overall, these reports conclude an insufficient benefit from surgery to justify the risk or the cost of 
DBS, a view reflected in our specific case [11]. However, as DBS is considered most effective in DOPA-responsive parkinsonism, and a subset of MSA cases will be responsive, it is interesting to consider why DBS fails in this group. It is possible that as MSA displays a wide, differential pattern of neurodegeneration compared to IPD, disease progression may lead to interruption of specific basal ganglia circuits ordinarily exploited successfully by DBS in IPD.

Nonetheless, DBS may retain a role in MSA in the absence of better treatment options. A small case series of 4 patients with Parkinson-predominant MSA unresponsive to L-DOPA did experience a small benefit in akinesia and rigidity with bilateral STN stimulation as our patient did. It produced a 10-15\% improvement in Activities of Daily Living scores maintained at 1 year after stimulation [12]. In the absence of a viable drug treatment, DBS could be considered, although the high cost of benefit to quality of life obtained makes DBS prohibitively expensive in MSA in most healthcare systems.

\section{Disease Duration}

Of special interest in this case is the prolonged survival of the patient in light of neuropathologically confirmed MSA. Average survival in patients with MSA is ordinarily very low, a feature that distinguishes it from IPD. The mean duration from onset to death is reported as 6-9 years (often as little as a year), with few cases of survival times similar to our case being noted $[1,13]$. It is currently unknown which factors dictate speed of deterioration, though Petrovic et al. [13] postulate early autonomic features as a likely negative prognostic factor.

In retrospect, some clinical features may have pointed towards MSA over IPD, but none were sufficiently pronounced or specific to challenge the diagnosis. Owing to the 6-year gap between surgery and autopsy, by which time all areas of the brain including striatum, cerebellum and brain stem were involved, it was not possible to determine whether areas such as the vagal nucleus, nucleus ambiguus or other pontine nuclei had been involved initially. Nonetheless, while histopathological confirmation of MSA was attained in this patient, until an antemortem unequivocal diagnosis of IPD (or MSA) can be made, it is almost impossible to ensure against such treatment failures in selecting for DBS.

\section{Conclusion}

This case report supports conclusions in the current published literature that STN stimulation in patients with L-DOPA-responsive Parkinson-predominant MSA is inappropriate and should be considered a contraindication to DBS. Our patient reflects the potential for clinically indistinguishable features common to MSA and IPD, and the issue this poses in both diagnosis and surgical referrals.

\section{Acknowledgements}

T. Aziz is supported by grants from the UK Medical Research Council, Norman Collisson Foundation, Charles Wolfson Charitable Trust, Oxford Partnership Comprehensive Biomedical Research Centre, with funding from the Department of Health's NIHR Biomedical Research Centres funding scheme. 
Thavanesan et al.: Deep Brain Stimulation in Multiple System Atrophy Mimicking Idiopathic Parkinson's Disease

\section{Disclosure Statement}

The authors declare that they have no competing interests. No specific funding was received in the production of this work.

\section{References}

1 Wenning GK, Tison F, Ben Shlomo Y, Daniel SE, Quinn NP: Multiple system atrophy: a review of 203 pathologically proven cases. Mov Disord 1997;12:133-147.

-2 Kim H-J, Jeon BS, Lee J-Y, Yun JY, Kim YE, Paek SH: Young-onset multiple system atrophy. J Neurol Sci 2012;319:168-170.

-3 Acar F, Acar G, Bir LS, Gedik B, Oğuzhanoğlu A: Deep brain stimulation of the pedunculopontine nucleus in a patient with freezing of gait. Stereotact Funct Neurosurg 2011;89:214-219.

-4 Ullman M, Vedam-Mai V, Resnick AS, Yachnis AT, McFarland NR, Merritt S, et al: Deep brain stimulation response in pathologically confirmed cases of multiple system atrophy. Parkinsonism Relat Disord 2012;18:86-88.

5 Talmant V, Esposito P, Stilhart B, Mohr M, Tranchant C: Subthalamic stimulation in a patient with multiple system atrophy: a clinicopathological report (in French). Rev Neurol (Paris) 2006;162:363-370.

-6 Santens P, Patrick S, Vonck K, Kristl V, De Letter M, Miet DL, et al: Deep brain stimulation of the internal pallidum in multiple system atrophy. Parkinsonism Relat Disord 2006;12:181-183.

7 Huang Y, Garrick R, Cook R, O'Sullivan D, Morris J, Halliday GM: Pallidal stimulation reduces treatmentinduced dyskinesias in 'minimal-change' multiple system atrophy. Mov Disord 2005;20:1042-1047.

8 Lezcano E, Gómez-Esteban JC, Zarranz JJ, Alcaraz R, Atarés B, Bilbao G, et al: Parkinson's disease-like presentation of multiple system atrophy with poor response to STN stimulation: a clinicopathological case report. Mov Disord 2004;19:973-977.

-9 Chou KL, Forman MS, Trojanowski JQ, Hurtig HI, Baltuch GH: Subthalamic nucleus deep brain stimulation in a patient with levodopa-responsive multiple system atrophy. Case report. J Neurosurg 2004;100:553-556.

10 Tarsy D, Apetauerova D, Ryan P, Norregaard T: Adverse effects of subthalamic nucleus DBS in a patient with multiple system atrophy. Neurology 2003;61:247-249.

11 Shih LC, Tarsy D: Deep brain stimulation for the treatment of atypical parkinsonism. Mov Disord 2007;22:2149-2155.

12 Visser-Vandewalle V, Temel Y, Colle H, van der Linden C: Bilateral high-frequency stimulation of the subthalamic nucleus in patients with multiple system atrophy-parkinsonism. Report of four cases. J Neurosurg 2003;98:882-887.

13 Petrovic IN, Ling H, Asi Y, Ahmed Z, Kukkle PL, Hazrati L-N, et al: Multiple system atrophy-parkinsonism with slow progression and prolonged survival: a diagnostic catch. Mov Disord 2012;27:1186-1190.

Table 1. Reports of DBS with MSA with DOPA-responsive symptoms

\begin{tabular}{llllll}
\hline $\begin{array}{l}\text { First author [ref.], } \\
\text { year }\end{array}$ & $\mathrm{n}$ & Indication for surgery & Target & Author conclusion & Survival after surgery \\
\hline Kim [2], 2012 & 2 & motor fluctuations, dyskinesia on DOPA & STN & negative & - \\
Acar [3], 2011 & 1 & gait freezing & PPN & positive & (6-month follow-up) \\
Ullman [4], 2012 & 2 & dyskinesia on medication (both patients) & STN & negative & 3 years, 4 months \\
Talmant [5], 2006 & 1 & on-off syndrome & STN & unfavourable & 2 years \\
Santens [6], 2006 & 1 & dystonic & GPi & negative & 7 months \\
Huang [7], 2005 & 1 & drug-induced dyskinesias & GPi & positive & - \\
Lezcano [8], 2004 & 1 & drug-induced dyskinesia, on-off syndrome & STN & negative & 18 months \\
Chou [9], 2004 & 1 & dyskinesia on medication, on-off syndrome & STN & negative & 12 weeks \\
Tarsy [10], 2003 & 1 & on-off syndrome, dyskinesia & STN & negative & - \\
\hline
\end{tabular}

PPN = Pedunculopontine nucleus; GPi = globus pallidus internus . 


\section{Case Reports in Neurology}
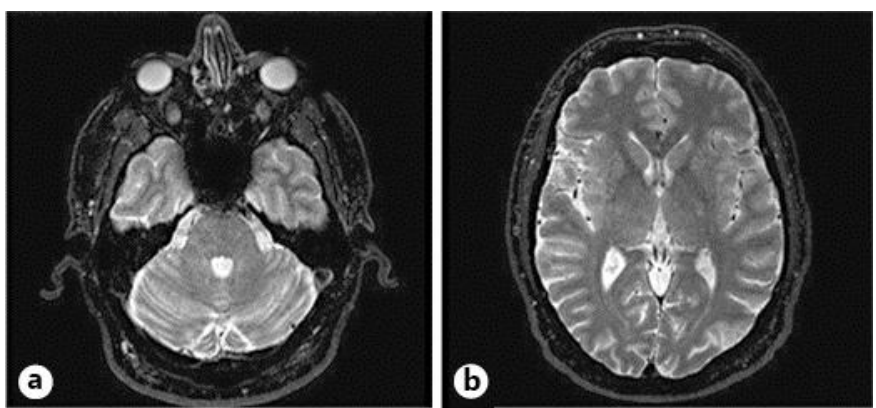

Fig. 1. T2-weighted MRI. a Axial section at the level of the mid-pons. b Axial section through the caudate, putamen, globus pallidus and thalamus.

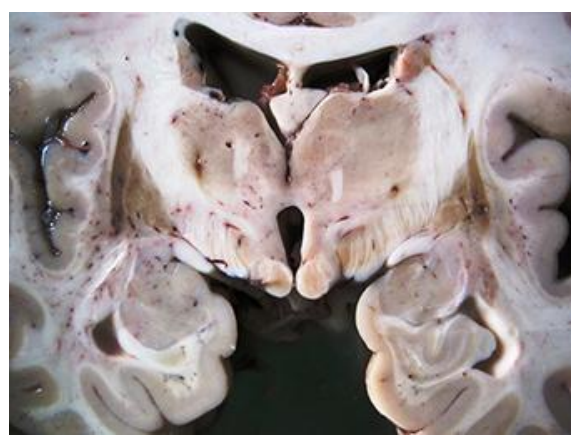

Fig. 2. Coronal section of the brain through the mammillary bodies to show sites of bilateral DBS insertion.

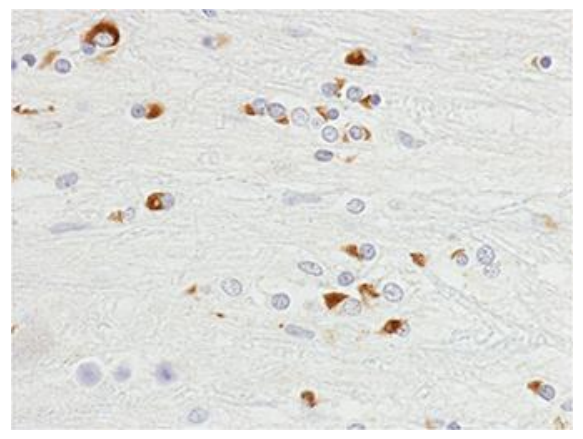

Fig. 3. Widespread oligodendroglial cytoplasmic $\alpha$-synuclein immunopositivity. 